\title{
Non-planar master integrals for the production of two off-shell vector bosons in collisions of massless partons
}

\author{
Fabrizio Caola, ${ }^{a}$ Johannes M. Henn, ${ }^{b}$ Kirill Melnikov ${ }^{a}$ and Vladimir A. Smirnov ${ }^{c}$ \\ ${ }^{a}$ Department of Physics and Astronomy, Johns Hopkins University, \\ Baltimore, U.S.A. \\ ${ }^{b}$ Institute for Advanced Study, \\ Princeton, NJ 08540, U.S.A. \\ ${ }^{c}$ Skobeltsyn Institute of Nuclear Physics of Moscow State University, \\ 119991 Moscow, Russia \\ E-mail: caola@pha.jhu.edu, jmhenn@ias.edu, melnikov@pha.jhu.edu, \\ smirnov@theory.sinp.msu.ru
}

ABSTRACT: We present the calculation of all non-planar master integrals that are needed to describe production of two off-shell vector bosons in collisions of two massless partons through NNLO in perturbative QCD. The integrals are computed analytically using differential equations in external kinematic variables and expressed in terms of Goncharov polylogarithms. These results provide the last missing ingredient needed for the computation of two-loop amplitudes that describe the production of two gauge bosons with different invariant masses in hadron collisions.

KeYwords: NLO Computations, Hadronic Colliders

ARXIV EPRINT: 1404.5590 


\section{Contents}

1 Introduction 1

2 Notation 2

3 Differential equations $\quad 4$

4 Solution in terms of multiple polylogarithms 5

$\begin{array}{lll}5 & \text { Master integrals } & 7\end{array}$

6 Conclusions $\quad 14$

\section{Introduction}

Perturbative QCD provides a viable framework to understand physics of hadron collisions. Continuous progress with perturbative QCD computations was instrumental for the success of the LHC physics program, crowned with the celebrated discovery of the Higgs boson. It is expected that a higher collision energy and the higher luminosity that will be reached when the LHC will resume its operations next year, will enable detailed studies of the multitude of various processes that involve elementary particles. It is therefore important to continue pushing frontiers of perturbative QCD in order to provide the best-possible theoretical predictions for relevant physics observables. A point in case is the production of two vector bosons, both on- and off-shell, in hadron collisions, $p p \rightarrow V_{1}^{*} V_{2}^{*}$. This process is interesting for a variety of physics reasons that we recently summarized in [1]. Considerations presented in [1] strongly motivate the extension of existing theoretical predictions for this process [29] to NNLO QCD. First and foremost, such an extension requires the scattering amplitude for a partonic processes $i j \rightarrow V_{1}^{*} V_{2}^{*}$ computed through two loops in perturbative QCD.

In ref. [1] we made a step towards the computation of this amplitude by calculating all two-loop planar integrals that contribute to these processes. ${ }^{1}$ The goal of the current paper is to complete the computation of the necessary ingredients for the two-loop amplitude calculation by providing explicit results for all relevant non-planar integrals. To compute them, we use the method of differential equations as suggested in ref. [11]. This allows us to choose the master integrals in such a way that iterative solution in the dimensional regularization parameter $\epsilon=(4-d) / 2$ becomes straightforward.

The remainder of the paper is organized as follows. In the next section, we introduce our notation and explain the basic strategy. In section 3 we discuss the differential

\footnotetext{
${ }^{1}$ Results for planar master integrals for the case of vector bosons with equal masses were first presented in $[10]$.
} 
equations and point out their general properties that are used later. In section 4 we explain how we constructed the analytic solutions of these differential equations in terms of multiple polylogarithms in the physical region. We also discuss how boundary conditions are computed. In section 5, we list non-planar master integrals and give their boundary asymptotic behavior in the physical region; we also present explicit results for divergences of some integrals and describe checks of our results. We conclude in section 6. Finally, in attached files, we give matrices that are needed to construct the differential equations for our basis of master integrals and the analytic results for all the non-planar two-loop threeand four-point integrals in terms of Goncharov polylogarithms.

\section{Notation}

We consider two-loop QCD corrections to the process $q\left(q_{1}\right) \bar{q}\left(q_{2}\right) \rightarrow V^{*}\left(q_{3}\right) V^{*}\left(q_{4}\right)$. The four-momenta of external particles satisfy $q_{1}^{2}=0, q_{2}^{2}=0$ and $q_{3}^{2}=M_{3}^{2}, q_{4}^{2}=M_{4}^{2}$. The Mandelstam invariants are $^{2}$

$$
S=\left(q_{1}+q_{2}\right)^{2}=\left(q_{3}+q_{4}\right)^{2}, \quad T=\left(q_{1}-q_{3}\right)^{2}=\left(q_{2}-q_{4}\right)^{2}, \quad U=\left(q_{1}-q_{4}\right)^{2}=\left(q_{2}-q_{3}\right)^{2} ;
$$

they satisfy the standard constraint $S+T+U=M_{3}^{2}+M_{4}^{2}$. The physical values of these kinematic variables are $M_{3}^{2}>0, M_{4}^{2}>0, S>\left(M_{3}+M_{4}\right)^{2}, T<0$ and $U<0$. Further constraints on these variables can be derived by considering the center-of-mass frame of colliding partons and expressing the transverse momentum of each of the vector bosons $\vec{q}_{\perp}$ through $T$ and $U$ variables. We find

$$
\vec{q}_{\perp}^{2}=\frac{\left(T U-M_{3}^{2} M_{4}^{2}\right)}{S} .
$$

In addition, the square of the three-momentum of each of the vector bosons in the centerof-mass frame reads

$$
\vec{q}^{2}=\frac{S^{2}-2 S\left(M_{3}^{2}+M_{4}^{2}\right)+\left(M_{3}^{2}-M_{4}^{2}\right)^{2}}{4 S} .
$$

The constraints on $T$ and $U$ for given $S, M_{3}^{2}, M_{4}^{2}$ follow from obvious inequalities

$$
0 \leq \vec{q}_{\perp}^{2} \leq \vec{q}^{2}
$$

All non-planar two-loop diagrams that are required for the production of two off-shell vector bosons can be described by a single meta-graph shown in figure 1 . Three mappings, that define three distinct families of integrals, need to be considered:

1. family $N_{12}: p_{1}=-q_{4}, p_{2}=-q_{3}, p_{3}=q_{2}, p_{4}=q_{1}$

2. family $N_{13}: p_{1}=-q_{4}, p_{2}=q_{2}, p_{3}=-q_{3}, p_{4}=q_{1}$;

3. family $N_{34}: p_{1}=q_{1}, p_{2}=q_{2}, p_{3}=-q_{3}, p_{4}=-q_{4}$.

\footnotetext{
${ }^{2}$ We use Mandelstam variables written with capital letters to refer to the physical process. Later, we will use Mandelstam variables for families of integrals; those we will write with lower case letters.
} 


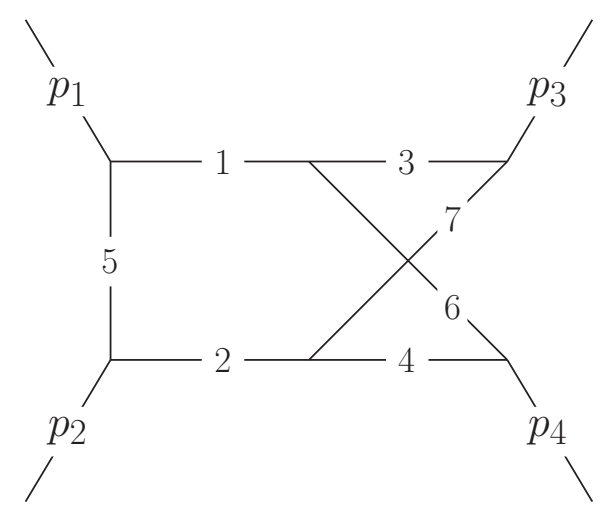

Figure 1. Double box non-planar graph. The numbering of the internal lines corresponds to the notation used in eqs. (2.5), (2.6). The ingoing external momenta satisfy $\sum_{i} p_{i}^{\mu}=0$. Different choices of on-shell conditions for them define the three non-planar integral families considered in the main text.

For each of these families, we define a set of integrals that is closed under the application of integration-by-parts identities. Specifically,

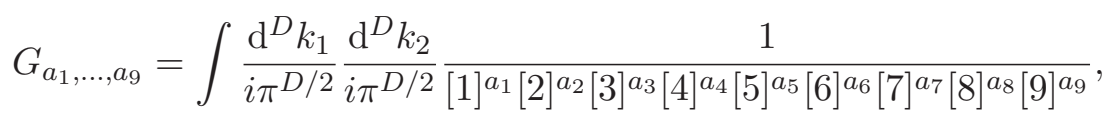

and

$$
\begin{aligned}
& {[1]=-k_{1}^{2}, \quad[2]=-\left(k_{1}+p_{1}+p_{2}\right)^{2}, \quad[3]=-k_{2}^{2},} \\
& {[4]=-\left(k_{1}-k_{2}+p_{1}+p_{2}+p_{3}\right)^{2}, \quad[5]=-\left(k_{1}+p_{1}\right)^{2}, \quad[6]=-\left(k_{1}-k_{2}\right)^{2},} \\
& {[7]=-\left(k_{2}-p_{3}\right)^{2}, \quad[8]=-\left(k_{2}+p_{1}\right)^{2}, \quad[9]=-\left(k_{1}-p_{3}\right)^{2} \text {. }}
\end{aligned}
$$

The exponents can take any integer values, with the restriction that $a_{8} \leq 0$ and $a_{9} \leq 0$. For each of the three families, integration-by-parts identities can be used to express all the integrals of that type through a minimal set of (master) integrals. Our choice of master integrals can be found in ancillary files. Many of these master integrals are, in fact, planar and were computed by some of us in ref. [1]. Genuine non-planar master integrals for each of the three families are shown and discussed in section 5 .

All master integrals satisfy differential equations in the external kinematic variables. These differential equations can be simplified by choosing suitable parametrizations of kinematic invariants, which removes square roots present in the original variables. For the families $N_{12}$ and $N_{13}$ we choose the parametrization to be

$$
S=M^{2}(1+x)(1+x y), \quad T=-M^{2} x z, \quad M_{3}^{2}=M^{2}, \quad M_{4}^{2}=M^{2} x^{2} y,
$$

where $M^{2}$ is the overall scale parameter. We note that the above parametrization is the same as in the planar case [1] and that in terms of the variables $x, y, z$, the physical region corresponds to

$$
x>0, \quad y>0, \quad y<z<1 .
$$


For the family $N_{34}$, the above parametrization can also be used but it is not optimal since it leads to the appearance of multiple terms $d \log (\alpha)$ in the differential equations where the letters $\alpha$ are quadratic in $x, y$ and $z$ which is problematic for the construction of an analytic solution that is based on Goncharov polylogarithms. Instead, we find it useful to choose the following parametrization

$$
\begin{array}{rlrl}
S & =M^{2}(1+x)^{2}, & T & =-M^{2} x((1+y)(1+x y)-2 z y(1+x)), \\
M_{3}^{2} & =M^{2} x^{2}\left(1-y^{2}\right), \quad M_{4}^{2} & =M^{2}\left(1-x^{2} y^{2}\right) .
\end{array}
$$

While the above parametrization also does not lead to a linear alphabet, it allows us to construct solutions in terms of Goncharov polylogarithms, as we explain below. ${ }^{3}$

\section{Differential equations}

In this section we describe a procedure [11] that allows us to compute the master integrals and comment on some aspects that arise when this procedure is applied to the calculation of non-planar integrals. We begin by deriving systems of differential equations for each of the above families. This is a relatively standard procedure, see e.g. refs. [12, 13], and we do not discuss it further. When deriving differential equations we performed a reduction to master integrals using the c++ version of program FIRE $[14,15]$. We choose all master integrals to be dimensionless, such that they depend only on the three variables $x, y, z$. Moreover, we choose them according to the procedure outlined in ref. [11] and find the simple form suggested in that references, namely

$$
\partial_{\xi} \vec{f}=\epsilon A_{\xi} \vec{f}
$$

where $\xi=x, y, z$ and $\vec{f}$ is a vector of master integrals. The master integrals for all the three families can be found in ancillary files; some examples of master integrals are discussed in section 5 .

The matrices $A_{\xi}$ contain simple rational functions (in $x, y, z$, see below). They satisfy the integrability conditions

$$
\left(\partial_{\xi} \partial_{\eta}-\partial_{\eta} \partial_{\xi}\right) \vec{f}=0 \quad \Rightarrow \quad \partial_{\xi} A_{\eta}-\partial_{\eta} A_{\xi}=0, \quad\left[A_{\eta}, A_{\xi}\right]=0,
$$

for $\xi, \eta \in\{x, y, z\}$. The structure of the equations can be further clarified by writing them in the combined form

$$
d \vec{f}(x, y, z ; \epsilon)=\epsilon d \tilde{A}(x, y, z) \vec{f}(x, y, z ; \epsilon),
$$

where the differential $d$ acts on $x, y$ and $z$. Matrices $\tilde{A}$ for each of the three families can be found in the ancillary files as well. For our choice of master integrals, the matrix $\tilde{A}$ can be written in the following way

$$
\tilde{A}=\sum_{i=1}^{N_{\max }} \tilde{A}_{\alpha_{i}} \log \left(\alpha_{i}\right),
$$

\footnotetext{
${ }^{3}$ We note that it is possible to obtain a linear alphabet for the $N_{34}$ family by changing variables $x \rightarrow x / y$ in eq. (2.9).
} 
where the $\tilde{A}_{\alpha_{i}}$ are constant matrices, and the arguments of the logarithms $\alpha_{i}$, called letters, are simple functions of $x, y, z$. The length of the alphabet $N_{\max }$ depends on the integral family. For families $N_{12}$ and $N_{13}$, we find the alphabet to be ${ }^{4}$

$$
\begin{aligned}
\alpha_{N_{12} \& N_{13}}= & \{x, 1+x, 1-y, y, 1+x y, 1-z, z, 1+y-z, z-y, \\
& 1+y+x y-z, x y+z, 1+x z, 1+x+x y-x z \\
& \left.z-y+y z+x y z, 1+y+2 x y-z+x^{2} y z\right\}
\end{aligned}
$$

For the family $N_{34}$, the alphabet reads

$$
\begin{aligned}
\alpha_{N_{34}}= & \{x, 1+x, 1-y, y, 1+y, 1-x y, 1+x y, 1-y(1-2 z), 1+y(1-2 z), \\
& 1-x y^{2}-(1-x) y(1-2 z), 1-x y(1-2 z), 1+x y(1-2 z), \\
& 1+x y^{2}-(1+x) y(1-2 z), 1-z, z, 1+y-2 y z, 1+y+x y(1+y-2 z)-2 y z, \\
& \left.1-y+2 y z, 1-x y^{2}+y(1-x-2 z+2 x z)\right\} .
\end{aligned}
$$

There are two things to be said about these alphabets for non-planar families. First, in contrast to planar master integrals, these alphabets contain quadratic polynomials. However, thanks to the chosen parametrization, for each integral family there is just one variable ( $x$ for $N_{12}, N_{13}$ and $y$ for $N_{34}$ ) with respect to which a particular alphabet is quadratic. Constructing explicit solutions for non-planar integrals requires integrating these alphabets over $x, y$ and $z$. This is not easy to do if quadratic polynomials need to be integrated. Nevertheless, it turns out that these alphabets can be integrated without much trouble provided that we postpone integration over quadratic variables until the very end of the calculation. Using this approach, integration can easily be performed in terms of Goncharov polylogarithms. We discuss this in more detail in the next section.

Second, we note that in the physical region, alphabets in eqs. (3.5), (3.6) are signdefinite. This feature implies that all iterated integrals needed for calculating $\vec{f}$ can be written in a manifestly real form, so that imaginary parts appear only through explicit factors of $i$. The latter come from the boundary conditions when they are computed in the physical region. This feature is similar to the case of planar master integral recently discussed in [1].

\section{Solution in terms of multiple polylogarithms}

In this section we review the procedure that allows us to solve the differential equations for the master integrals, following the discussion in our previous paper [1]. The vector of master integrals $\vec{f}$ can be expanded in powers of $\epsilon$,

$$
\vec{f}=\sum_{i=0}^{4} \vec{f}^{(i)} \epsilon^{i}+\mathcal{O}\left(\epsilon^{5}\right) .
$$

To construct a solution of the differential equation, we need to iteratively solve eq. (3.1) order-by-order in the dimensional-regularization parameter $\epsilon$. Suppose the solution is constructed up to $i=n-1$. The set of differential equations for $\vec{f}^{(n)}$ is then

$$
\partial_{x} \vec{f}^{(n)}=A_{x} \vec{f}^{(n-1)}, \quad \partial_{y} \vec{f}^{(n)}=A_{y} \vec{f}^{(n-1)}, \quad \partial_{z} \vec{f}^{(n)}=A_{z} \vec{f}^{(n-1)} .
$$

\footnotetext{
${ }^{4}$ First fourteen letters in eq. (3.5) give the alphabet for $N_{12}$.
} 
To find $\vec{f}^{(n)}$, we integrate the first equation over $x$; substitute the solution back to the differential equation for $y$, integrate again, substitute the solution back into the differential equation for $z$ and integrate again. This procedure determines $\vec{f}^{(n)}$ up to a constant of integration that is then fixed from boundary conditions.

To solve the differential equations in eq. (4.2), we should be able to integrate inverse elements of the alphabets that we displayed in the previous section. Since, as we pointed out already, elements of the alphabet can be both linear and quadratic in certain variables, such integration appears to be more complicated than the case of a linear alphabet that always permits to write a solution in terms of Goncharov polylogarithms,

$$
G\left(a_{n}, a_{n-1}, \ldots . a_{1}, t\right)=\int_{0}^{t} \frac{\mathrm{d} t_{n}}{t_{n}-a_{n}} G\left(a_{n-1}, \ldots . a_{1}, t_{n}\right) .
$$

This concern is, however, unfounded and solution in terms of Goncharov polylogarithms can be constructed for the alphabets of families $N_{12}, N_{13}$ and $N_{34}$ as well. To show this, we consider, for definiteness, the alphabet of the family $N_{34}$ which is quadratic in $y$ but is linear in $x$ and $z$. Suppose we choose to integrate over $z$ first, over $x$ second, and, finally, over $y$. One of the quadratic letters of the alphabet $\alpha_{34}$ reads $1+x y^{2}-(1+x) y(1-2 z)$. Upon integrating it over $x$, we obtain Goncharov polylogarithms of the form $G[(1-y+$ $2 y z) /(y(1-y-2 z)), \ldots, x]$. When this solution is used to derive the differential equation for the function of the other two variables $z, y$, all functions that depend on $x$ should cancel out. This implies that, by the time we get to the $y$-integration, all the letters of the alphabet that are quadratic in $y$ and, at the same time, depend on $z$ and $x$ should disappears. Since there are no letters in the alphabet that are quadratic in $y$ and are independent of $x$ and $z$, we conclude that only letters of the alphabet that are linear in $y$ appear at the final stage of the integration. A similar consideration shows that, in case of the family $N_{13}$, we can avoid the need to deal with quadratic letters of the alphabet provided that we first integrate over $y$, then over $z$ and, finally, over $x$.

A full construction of analytic solutions requires boundary conditions and we compute them directly in the physical region. Because of the different parametrizations used for different integral families, boundary conditions are obtained from different limits of $x, y$ and $z$ variables. For families $N_{12}, N_{13}$, we consider the limits $x \rightarrow 0, y \rightarrow z, z \rightarrow 1$, and for the family $N_{34}$, the limit $x \rightarrow 0, y \rightarrow 0$ and $z \rightarrow 1$. Note that the physical meaning of these limits corresponds to the kinematic situation where the colliding partons have just enough energy to produce two vector bosons with very different masses $M_{i}^{2} \ll M_{j}^{2} \sim S$. In this limit, the absolute value of the three-momenta of vector bosons vanishes and the scattering occurs in the forward direction. This is the same kinematics that we used in our previous paper on planar master integrals [1] and, since many planar integrals appear in the current computation as non-homogeneous terms in the differential equations, the boundary conditions computed in [1] can be recycled for a large number of required integrals.

The boundary conditions are a priori unknown for genuine non-planar integrals and we compute them in two different ways. One possibility is to study consistency conditions of differential equations in three variables; this procedure is discussed in ref. [1] and it is often 
sufficient to fix the required boundary behavior of non-planar integrals. Another possibility is to compute the relevant limits directly, expanding Feynman integrals in small kinematic variables. To accomplish this, we used the strategy of expansion by regions $[16,17]$ (for a recent review see chapter 9 of ref. [18]) and its implementation in the public computer code asy.m $[19,20]$ which is now included into FIESTA [21].

\section{Master integrals}

For each family of integrals, the Mandelstam variables are given by $s=\left(p_{1}+p_{2}\right)^{2}=$ $\left(p_{3}+p_{4}\right)^{2}, t=\left(p_{1}+p_{3}\right)^{2}=\left(p_{2}+p_{4}\right)^{2}, u=\left(p_{2}+p_{3}\right)^{2}=\left(p_{1}+p_{3}\right)^{2}$. Their relation to the physical Mandelstam variables $S, T, U$ and the ensuing parametrization in terms of variables $x, y, z$ can be read off using the $q \rightarrow p$ mapping just before eq. (2.5) and eqs. (2.1), (2.7).

We choose the master integrals following the strategy suggested in ref. [11]. The idea is to find master integrals whose Laurent expansion in $\epsilon$ leads to expressions of a uniform weight. As guiding principles for finding such integrals, we analyzed generalized unitarity cuts, as well as explicit (Feynman) parameter representations of the integrals. Technically this is very similar to the analysis of certain three-loop massless integrals studied in refs. [22, 23]. In fact, some of the two-loop integrals with two off-shell legs are contained in those three-loop integrals as subintegrals. For more detailed explanations and examples, see section 2 of ref. [22]. In ref. [24] the problem of choosing suitable master integrals was related to the diagonalization of matrices $\tilde{A}$.

Below we present the master integrals and the boundary conditions in the physical region. For all the three families we choose the master integrals to be $f_{i}=N_{0} M^{4 \epsilon} e^{2 \gamma_{\mathrm{E}} \epsilon} g_{i}$, where $M$ is the overall mass-dimension scaling parameter used to parametrize Mandelstam invariants. The normalization constant $N_{0}$ is

$$
N_{0}=1+\frac{\pi^{2}}{6} \epsilon^{2}+\frac{32 \zeta_{3}}{3} \epsilon^{3}+\frac{67 \pi^{4} \epsilon^{4}}{360} .
$$

Furthermore, to present the master integrals and the results for the limits, we use the following notation

$$
\begin{array}{rlrl}
N_{3} & =1-i \epsilon \pi-\frac{\pi^{2} \epsilon^{2}}{6}-\left(\frac{i \pi^{3}}{6}+14 \zeta_{3}\right) \epsilon^{3}, & R_{12} & =\sqrt{p_{1}^{2}+\left(p_{2}^{2}-s\right)^{2}-2 p_{1}^{2}\left(p_{2}^{2}+s\right)}, \\
R_{13}=\sqrt{p_{1}^{2}+\left(p_{3}^{2}-t\right)^{2}-2 p_{1}^{2}\left(p_{3}^{2}+t\right)}, & R_{34}=\sqrt{p_{3}^{2}+\left(p_{4}^{2}-s\right)^{2}-2 p_{3}^{2}\left(p_{4}^{2}+s\right)} .
\end{array}
$$

In contrast to our previous paper [1], we will not present results for all integrals that are needed to construct the non-planar master integrals. The reason is that many of these integrals are the planar ones; they were computed in ref. [1]. For the family $N_{34}$, some of the planar integrals need to be re-expressed in new variables, since the parametrization of the family $N_{34}$ differs from the parametrization used for all other families. This is straightforward to do, at least in principle. Therefore, below we present our choices of the genuine nonplanar integrals and the boundary conditions for them. However, we note that a complete set of all master integrals for the three integral families can be found in the ancillary files. 
Finally, we note that the pictures of master integrals shown below are intended to give a general idea of how the corresponding master integrals look like, but they do not show squared propagators, numerators and prefactors. Also, in some cases we chose linear combinations of integrals as master integrals, and in those cases only one representative figure is given.

For the family $N_{12}$, there are eight genuine non-planar master integrals. The boundary conditions are evaluated at the point $x \rightarrow 0, y \rightarrow 1, z \rightarrow 1$. More specifically, we write $x=\delta, y=1-2 \delta$ and $z=1-\delta$ and consider the $\delta \rightarrow 0$ limit. In the following, we indicate with ' $\sim$ ' an equality up to power-suppressed terms in $\delta$.
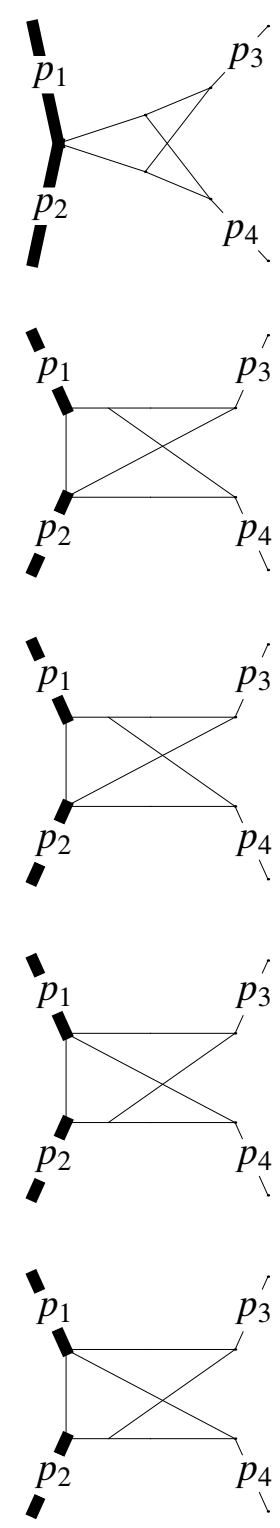

$$
\begin{aligned}
g_{28}^{\mathrm{N} 12} & =\epsilon^{4} s^{2} G_{1,1,1,1,0,1,1,0,0} \\
f_{28}^{\mathrm{N} 12} & \sim e^{2 i \pi \epsilon}\left(1-\frac{5 \pi^{2} \epsilon^{2}}{6}-17 \zeta_{3} \epsilon^{3}-\frac{17 \pi^{4} \epsilon^{4}}{36}\right),
\end{aligned}
$$$$
g_{29}^{\mathrm{N} 12}=\epsilon^{2} p_{1}^{2} s G_{1,0,1,1,1,1,1,0,0},
$$$$
f_{29}^{\mathrm{N} 12} \sim \frac{x^{-4 \epsilon}}{4}\left[1+10 i \pi \epsilon-\frac{46 \pi^{2} \epsilon^{2}}{3}-\left(12 i \pi^{3}-16 \zeta_{3}\right) \epsilon^{3}\right.
$$$$
\left.+\left(\frac{386 \pi^{4}}{45}-32 i \pi \zeta_{3}\right) \epsilon^{4}\right]-i \pi \epsilon x^{-4 \epsilon}[(z-y)(1-z)]^{-2 \epsilon},
$$$$
g_{30}^{\mathrm{N} 12}=\epsilon^{4}\left(\left(-p_{2}^{2}+t\right) G_{0,0,1,1,1,1,1,0,0}+\left(u-p_{1}^{2}\right) G_{1,0,1,1,1,1,1,0,-1}\right),
$$$$
f_{30}^{\mathrm{N} 12} \sim \frac{1}{4} e^{2 i \pi \epsilon}-\frac{1}{4} x^{-2 \epsilon}+x^{-4 \epsilon}\left[-i \pi \epsilon+\frac{10 \pi^{2} \epsilon^{2}}{6}\right.
$$$$
\left.+\left(\frac{4 i \pi^{3}}{3}-2 \zeta_{3}\right) \epsilon^{3}-\left(\frac{89 \pi^{4}}{90}-4 i \pi \zeta_{3}\right) \epsilon^{4}\right],
$$

$$
\begin{aligned}
g_{31}^{\mathrm{N} 12}= & \epsilon^{4} p_{2}^{2} s G_{0,1,1,1,1,1,1,0,0} \\
f_{31}^{\mathrm{N} 12} \sim & 1+2 i \pi \epsilon-\frac{17 \pi^{2} \epsilon^{2}}{6}-\left(3 i \pi^{3}+17 \zeta_{3}\right) \epsilon^{3}+\left(\frac{67 \pi^{4}}{36}-34 i \pi \zeta_{3}\right) \epsilon^{4} \\
& -x^{-2 \epsilon}\left(1-\frac{\pi^{2} \epsilon^{2}}{6}-7 \zeta_{3} \epsilon^{3}-\frac{\pi^{4} \epsilon^{4}}{3}\right)+\frac{1}{4} e^{2 i \pi \epsilon} x^{-4 \epsilon} \\
& -i \pi \epsilon x^{-4 \epsilon}[(z-y)(1-z)]^{-2 \epsilon}, \\
g_{32}^{\mathrm{N} 12}= & \epsilon^{4}\left(\left(t-p_{1}^{2}\right) G_{0,0,1,1,1,1,1,0,0}+\left(p_{1}^{2}-s-t\right) G_{0,1,1,1,1,1,1,0,-1}\right), \\
f_{32}^{\mathrm{N} 12} \sim & -\frac{1}{4}-\frac{i \pi \epsilon}{2}+\frac{11 \pi^{2} \epsilon^{2}}{12}+\left(\frac{7 i \pi^{3}}{6}+\frac{17 \zeta_{3}}{2}\right) \epsilon^{3}-\left(\frac{55 \pi^{4}}{72}-17 i \pi \zeta_{3}\right) \epsilon^{4} \\
& +x^{-2 \epsilon}\left(\frac{1}{4}-\frac{\pi^{2} \epsilon^{2}}{12}-\frac{7 \zeta_{3} \epsilon^{3}}{2}-\frac{\pi^{4} \epsilon^{4}}{6}\right)
\end{aligned}
$$




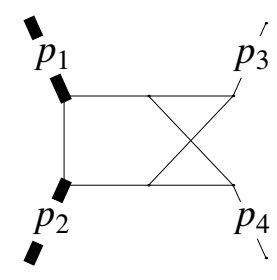

$$
\begin{aligned}
g_{33}^{\mathrm{N} 12}= & \epsilon^{4} s t\left[G_{0,1,1,1,1,1,1,0,0}+G_{1,0,1,1,1,1,1,0,0}\right. \\
& \left.-G_{1,1,1,1,1,1,1,-1,0}+s G_{1,1,1,1,1,1,1,0,0}\right], \\
f_{33}^{\mathrm{N} 12} \sim & -\frac{x^{-2 \epsilon}}{2}\left(1-\frac{\pi^{2} \epsilon^{2}}{6}-7 \zeta_{3} \epsilon^{3}-\frac{\pi^{4} \epsilon^{4}}{3}\right) \\
+ & \frac{x^{-4 \epsilon}}{4}\left(1+6 i \pi \epsilon-\frac{26 \pi^{2} \epsilon^{2}}{3}+\left(8 \zeta_{3}-\frac{20 i \pi^{3}}{3}\right) \epsilon^{3}\right. \\
& \left.+\left(\frac{208 \pi^{4}}{45}-16 i \pi \zeta_{3}\right) \epsilon^{4}\right) \\
& -i \pi \epsilon x^{-4 \epsilon}[(z-y)(1-z)]^{-2 \epsilon},
\end{aligned}
$$

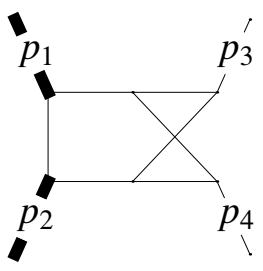

$$
\begin{aligned}
g_{34}^{\mathrm{N} 12} & =-\epsilon^{4} s u G_{1,1,1,1,1,1,1,-1,0}, \\
f_{34}^{\mathrm{N} 12} & \sim \frac{x^{-2 \epsilon}}{2}\left(1-\frac{\pi^{2} \epsilon^{2}}{6}-7 \zeta_{3} \epsilon^{3}-\frac{\pi^{4} \epsilon^{4}}{3}\right)
\end{aligned}
$$$$
-\frac{x^{-4 \epsilon}}{4}\left(1+6 i \pi \epsilon-\frac{26 \pi^{2} \epsilon^{2}}{3}+\left(8 \zeta_{3}-\frac{20 i \pi^{3}}{3}\right) \epsilon^{3}\right.
$$$$
\left.+\left(\frac{208 \pi^{4}}{45}-16 i \pi \zeta_{3}\right) \epsilon^{4}\right)
$$$$
+i \pi \epsilon x^{-4 \epsilon}[(z-y)(1-z)]^{-2 \epsilon} \text {, }
$$

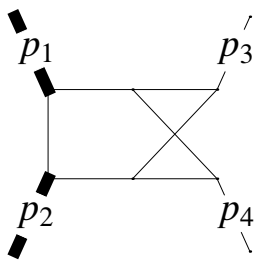

$$
\begin{aligned}
& g_{35}^{\mathrm{N} 12}=\epsilon^{4} R_{12}\left[G_{0,0,1,1,1,1,1,0,0}-G_{0,1,1,1,1,1,1,0,-1}\right. \\
& \left.-G_{1,0,1,1,1,1,1,0,-1}-s G_{1,1,1,1,1,1,1,-1,0}+G_{1,1,1,1,1,1,1,0,-2}\right], \\
& f_{35}^{\mathrm{N} 12} \sim 0
\end{aligned}
$$

There are nine non-planar master integrals in the family $N_{13}$. These integrals, together with their limits in the kinematic point $x \rightarrow 0, y \rightarrow 1, z \rightarrow 1$ are

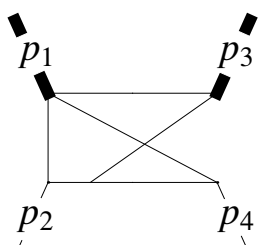

$$
\begin{aligned}
g_{33}^{\mathrm{N} 13}= & \epsilon^{4} p_{3}^{2} t G_{0,1,1,1,1,1,1,0,0} \\
f_{33}^{\mathrm{N} 13} \sim & 1+2 i \pi \epsilon-\frac{17 \pi^{2} \epsilon^{2}}{6}-\left(3 i \pi^{3}+17 \zeta_{3}\right) \epsilon^{3}+\left(\frac{67 \pi^{4}}{36}-34 i \pi \zeta_{3}\right) \epsilon^{4} \\
& -x^{-2 \epsilon}\left(1-\frac{\pi^{2} \epsilon^{2}}{6}-7 \zeta_{3} \epsilon^{3}-\frac{\pi^{4} \epsilon^{4}}{3}\right)+\frac{e^{2 i \pi \epsilon}}{4} x^{-4 \epsilon} \\
& -i \pi \epsilon x^{-4 \epsilon}[(z-y)(1-z)]^{-2 \epsilon}, \\
g_{34}^{\mathrm{N} 13}= & \epsilon^{4}\left(\left(t-p_{1}^{2}+p_{3}^{2}\right) G_{0,0,1,1,1,1,1,0,0}+\left(u-p_{3}^{2}\right) G_{0,1,1,1,1,1,1,0,-1}\right), \\
f_{34}^{\mathrm{N} 13} \sim & \frac{1}{2}+i \pi \epsilon-\frac{3 \pi^{2} \epsilon^{2}}{2}-\left(\frac{5 i \pi^{3}}{3}+9 \zeta_{3}\right) \epsilon^{3}+\left(\frac{193 \pi^{4}}{180}-18 i \pi \zeta_{3}\right) \epsilon^{4} \\
& -x^{-2 \epsilon}\left(\frac{3}{4}-\frac{\pi^{2} \epsilon^{2}}{4}-\frac{21 \zeta_{3} \epsilon^{3}}{2}-\frac{\pi^{4} \epsilon^{4}}{2}\right) \\
& +x^{-4 \epsilon}\left(\frac{1}{4}+\frac{i \pi \epsilon}{2}-\frac{\pi^{2} \epsilon^{2}}{2}-\frac{i \pi^{3} \epsilon^{3}}{3}+\frac{\pi^{4} \epsilon^{4}}{6}\right) \\
& -i \pi \epsilon x^{-4 \epsilon}[(z-y)(1-z)]^{-2 \epsilon},
\end{aligned}
$$

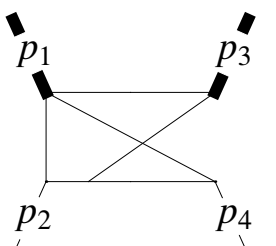



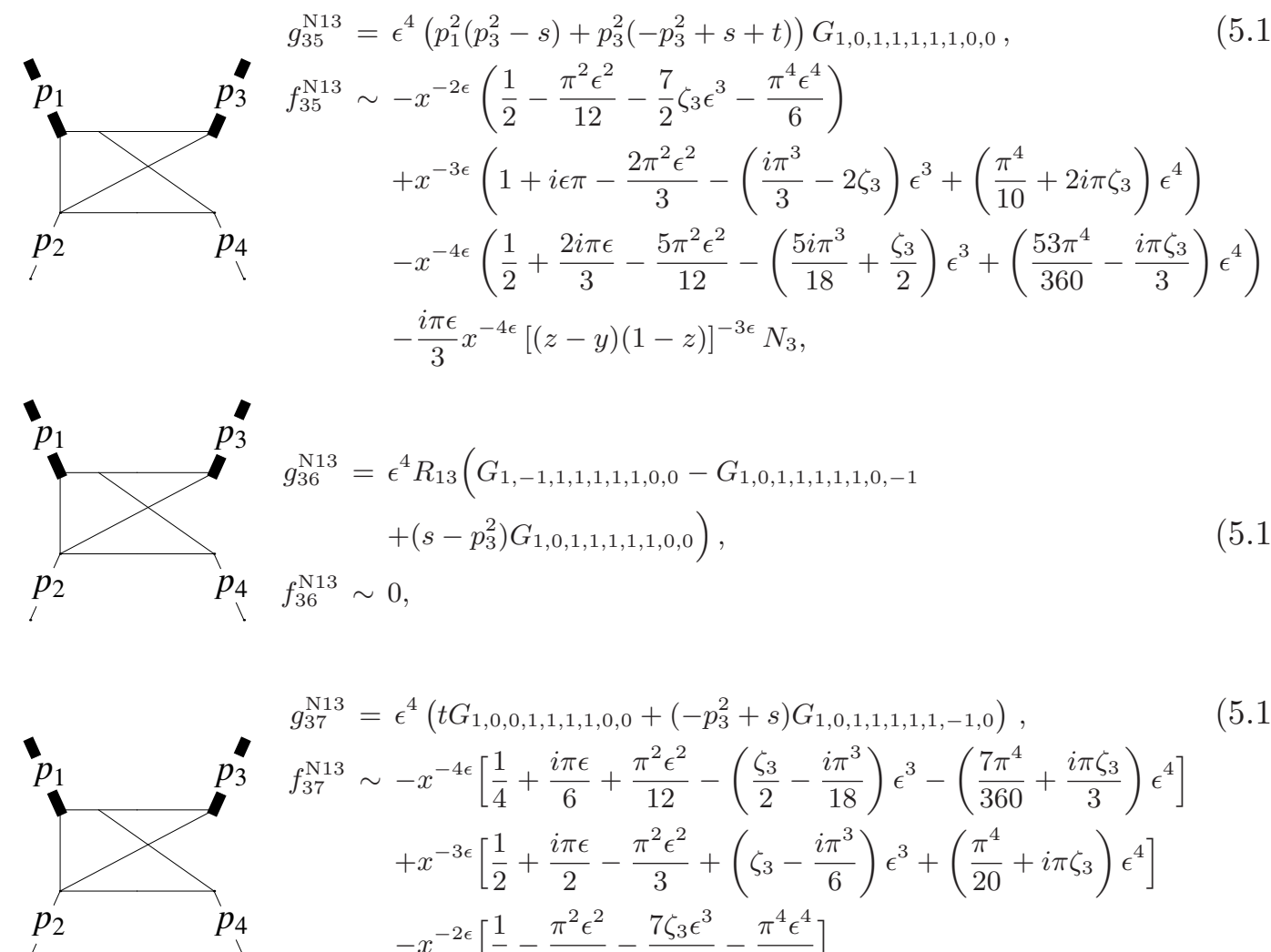

$$
g_{37}^{\mathrm{N} 13}=\epsilon^{4}\left(t G_{1,0,0,1,1,1,1,0,0}+\left(-p_{3}^{2}+s\right) G_{1,0,1,1,1,1,1,-1,0}\right)
$$

$f_{37}^{\mathrm{N} 13} \sim-x^{-4 \epsilon}\left[\frac{1}{4}+\frac{i \pi \epsilon}{6}+\frac{\pi^{2} \epsilon^{2}}{12}-\left(\frac{\zeta_{3}}{2}-\frac{i \pi^{3}}{18}\right) \epsilon^{3}-\left(\frac{7 \pi^{4}}{360}+\frac{i \pi \zeta_{3}}{3}\right) \epsilon^{4}\right]$

$+x^{-3 \epsilon}\left[\frac{1}{2}+\frac{i \pi \epsilon}{2}-\frac{\pi^{2} \epsilon^{2}}{3}+\left(\zeta_{3}-\frac{i \pi^{3}}{6}\right) \epsilon^{3}+\left(\frac{\pi^{4}}{20}+i \pi \zeta_{3}\right) \epsilon^{4}\right]$

$-x^{-2 \epsilon}\left[\frac{1}{4}-\frac{\pi^{2} \epsilon^{2}}{12}-\frac{7 \zeta_{3} \epsilon^{3}}{2}-\frac{\pi^{4} \epsilon^{4}}{6}\right]$

$-\frac{i \epsilon \pi}{3} x^{-4 \epsilon}[(z-y)(1-z)]^{-3 \epsilon} N_{3}$,

$$
f_{38}^{\mathrm{N} 13}=\epsilon^{4}\left(-t G_{0,0,1,1,1,1,1,0,0}+\left(-p_{3}^{2}+s+t\right) G_{1,0,1,1,1,1,1,0,-1}\right),
$$

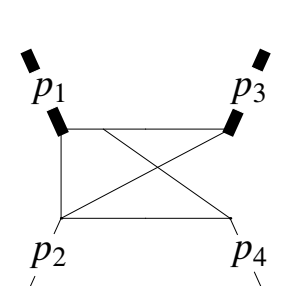

$$
\begin{aligned}
f_{38}^{\mathrm{N} 13} \sim & \frac{\pi^{2} \epsilon^{2}}{12}+\epsilon^{3}\left(\frac{\zeta_{3}}{2}+\frac{i \pi^{3}}{6}\right)-\epsilon^{4}\left(\frac{17 \pi^{4}}{120}-i \pi \zeta_{3}\right) \\
& +x^{-2 \epsilon}\left[\frac{1}{2}-\frac{\pi^{2} \epsilon^{2}}{12}-\frac{7 \zeta_{3} \epsilon^{3}}{2}-\frac{\pi^{4} \epsilon^{4}}{6}\right] \\
& -x^{-3 \epsilon}\left[1+i \pi \epsilon-\frac{2 \pi^{2} \epsilon^{2}}{3}+\left(2 \zeta_{3}-\frac{i \pi^{3}}{3}\right) \epsilon^{3}+\left(\frac{\pi^{4}}{10}+2 i \pi \zeta_{3}\right) \epsilon^{4}\right] \\
& +x^{-4 \epsilon}\left[\frac{1}{2}+\frac{2 i \pi \epsilon}{3}-\frac{5 \pi^{2} \epsilon^{2}}{12}-\left(\frac{\zeta_{3}}{2}+\frac{5 i \pi^{3}}{18}\right) \epsilon^{3}+\left(\frac{53 \pi^{4}}{360}-\frac{i \pi \zeta_{3}}{3}\right) \epsilon^{4}\right] \\
& +\frac{i \epsilon \pi}{3} x^{-4 \epsilon}[(z-y)(1-z)]^{-3 \epsilon} N_{3},
\end{aligned}
$$

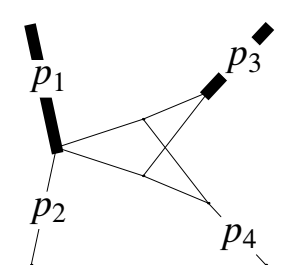

$$
\begin{aligned}
g_{40}^{\mathrm{N} 13}= & \epsilon^{4}\left(p_{3}^{2}-s\right)^{2} G_{1,1,1,1,0,1,1,0,0} \\
f_{40}^{\mathrm{N} 13} \sim & 1+2 i \pi \epsilon-\frac{17 \pi^{2} \epsilon^{2}}{6}-\epsilon^{3}\left(17 \zeta_{3}+3 i \pi^{3}\right)+\epsilon^{4}\left(\frac{67 \pi^{4}}{36}-34 i \pi \zeta_{3}\right) \\
& -x^{-\epsilon}\left[2+2 i \pi \epsilon-2 \pi^{2} \epsilon^{2}-\left(12 \zeta_{3}+\frac{4 i \pi^{3}}{3}\right) \epsilon^{3}+\left(\frac{\pi^{4}}{30}-12 i \pi \zeta_{3}\right) \epsilon^{4}\right] \\
& +x^{-2 \epsilon}\left[1-\frac{\pi^{2} \epsilon^{2}}{6}-7 \epsilon^{3} \zeta_{3}-\frac{\pi^{4} \epsilon^{4}}{3}\right]
\end{aligned}
$$




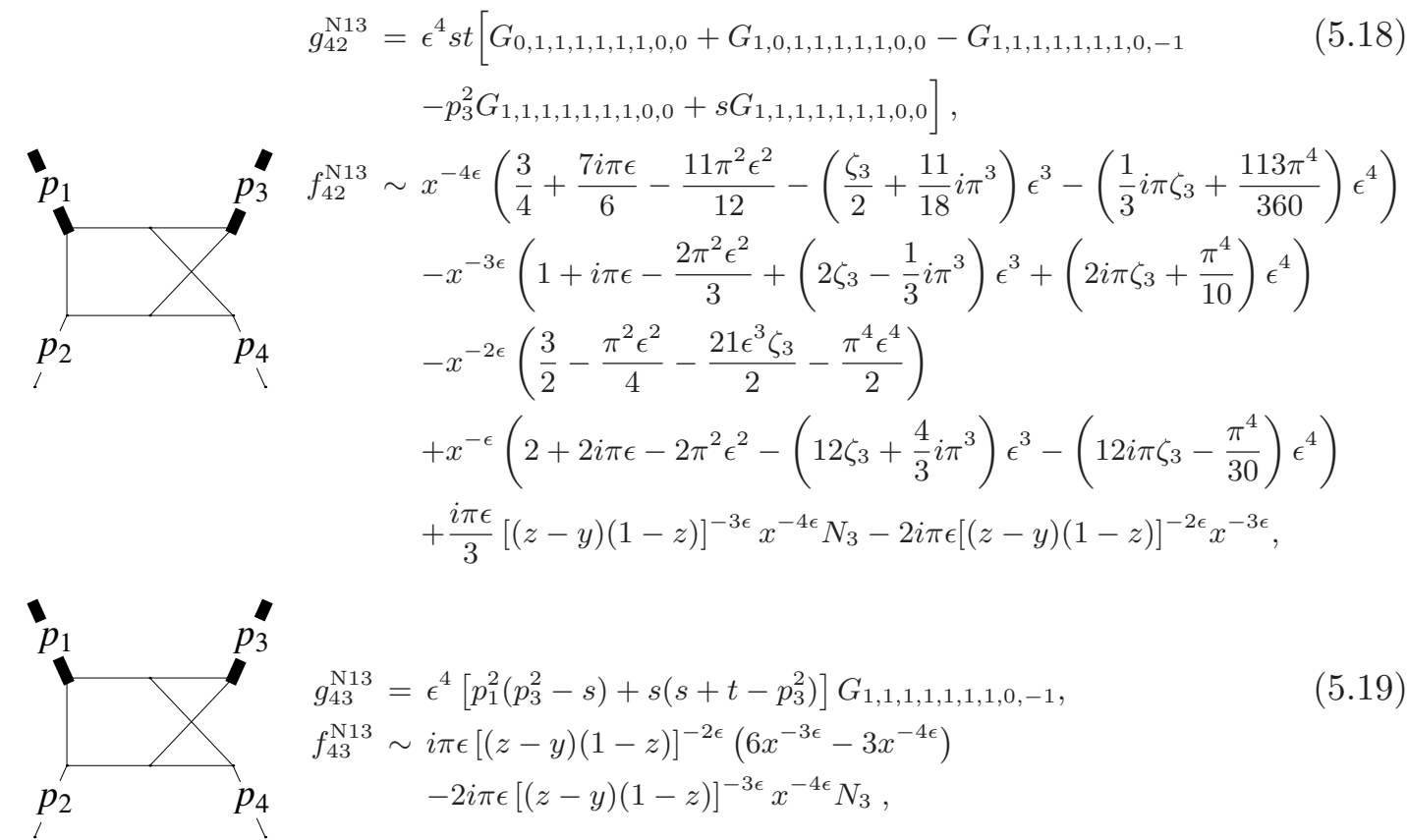

Non-planar master integrals that appear for the family $N_{34}$ are shown below. The boundary conditions are derived by considering the limit $x \rightarrow 0, y \rightarrow 0$ and $z \rightarrow 1$. The results read

$$
\begin{aligned}
g_{37}^{\mathrm{N} 34}= & \epsilon^{4}\left(p_{3}^{2}\left(p_{4}^{2}-t\right)+p_{4}^{2}\left(s+t-p_{4}^{2}\right)\right) G_{0,1,1,1,1,1,1,0,0}, \\
f_{37}^{\mathrm{N} 34} \sim & -x^{-4 \epsilon}\left[\frac{1}{2}+\frac{2 i \pi \epsilon}{3}-\frac{5 \pi^{2} \epsilon^{2}}{12}-\epsilon^{3}\left(\frac{\zeta_{3}}{2}+\frac{5 i \pi^{3}}{18}\right)-\epsilon^{4}\left(-\frac{53 \pi^{4}}{360}+\frac{i \pi \zeta_{3}}{3}\right)\right] \\
& +x^{-3 \epsilon}\left[1+i \pi \epsilon-\frac{2 \pi^{2} \epsilon^{2}}{3}+\epsilon^{3}\left(2 \zeta_{3}-\frac{i \pi^{3}}{3}\right)+\epsilon^{4}\left(\frac{\pi^{4}}{10}+2 i \pi \zeta_{3}\right)\right] \\
& -x^{-2 \epsilon}\left[\frac{1}{2}-\frac{\pi^{2} \epsilon^{2}}{12}-\frac{7 \epsilon^{3} \zeta_{3}}{2}-\frac{\pi^{4} \epsilon^{4}}{6}\right] \\
& -\frac{i \pi \epsilon}{3}\left[4 y^{2}(1-z)\right]^{-3 \epsilon} x^{-4 \epsilon} N_{3},
\end{aligned}
$$




$$
\begin{aligned}
g_{39}^{\mathrm{N} 34}= & \epsilon^{4}\left[-s G_{0,1,1,1,1,1,0,0,0}+\left(p_{4}^{2}-s-t\right)\left(-G_{0,0,1,1,1,1,1,0,0}\right.\right. \\
& +G_{0,1,1,0,1,1,1,0,0}-G_{0,1,1,1,1,0,1,0,0} \\
& \left.\left.-G_{0,1,1,1,1,1,0,0,0}+G_{0,1,1,1,1,1,1,0,-1}+p_{4}^{2} G_{0,1,1,1,1,1,1,0,0}\right)\right], \\
f_{39}^{\mathrm{N} 34} \sim & \frac{\pi^{2} \epsilon^{2}}{12}+\epsilon^{3}\left(\frac{\zeta_{3}}{2}+\frac{i \pi^{3}}{6}\right)+\epsilon^{4}\left(-\frac{17 \pi^{4}}{120}+i \pi \zeta_{3}\right) \\
+ & +x^{-4 \epsilon}\left(+\frac{1}{2}+\frac{2 i \pi \epsilon}{3}-\frac{5 \pi^{2} \epsilon^{2}}{12}+\epsilon^{3}\left(-\frac{\zeta_{3}}{2}-\frac{5 i \pi^{3}}{18}\right)+\epsilon^{4}\left(\frac{53 \pi^{4}}{360}-\frac{i \pi \zeta_{3}}{3}\right)\right) \\
& -x^{-3 \epsilon}\left(1+i \pi \epsilon-\frac{2 \pi^{2} \epsilon^{2}}{3}+\epsilon^{3}\left(2 \zeta_{3}-\frac{i \pi^{3}}{3}\right)+\epsilon^{4}\left(\frac{\pi^{4}}{10}+2 i \pi \zeta_{3}\right)\right) \\
+ & x^{-2 \epsilon}\left(\frac{1}{2}-\frac{\pi^{2} \epsilon^{2}}{12}-\frac{7 \epsilon^{3} \zeta_{3}}{2}-\frac{1}{6} \pi^{4} \epsilon^{4}\right) \\
+ & \frac{i \pi}{3} \epsilon\left[4 y^{2}(1-z)\right]^{-3 \epsilon} x^{-4 \epsilon} N_{3},
\end{aligned}
$$

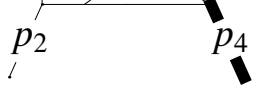

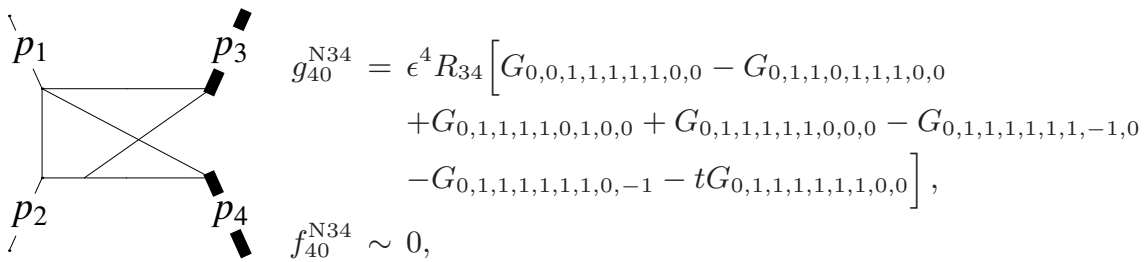

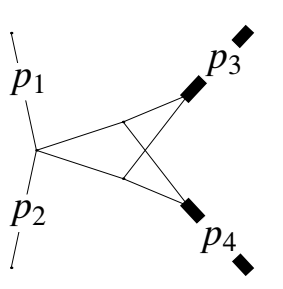

$$
\begin{aligned}
g_{47}^{\mathrm{N} 34} & =\epsilon^{4}\left(p_{3}^{2}+\left(p_{4}^{2}-s\right)^{2}-2 p_{3}^{2}\left(p_{4}^{2}+s\right)\right) G_{1,1,1,1,0,1,1,0,0} \\
f_{47}^{\mathrm{N} 34} & \sim 0
\end{aligned}
$$

$$
g_{50}^{\mathrm{N} 34}=\left[2\left(p_{3}^{2}+p_{4}^{2}-s\right)\right]^{-1} \epsilon^{4} s\left[-2 p_{3}^{2} p_{4}^{2} G_{0,1,1,1,1,1,1,0,0}-2 p_{3}^{2} p_{4}^{2} G_{1,0,1,1,1,1,1,0,0}\right.
$$$$
-\left(p_{3}^{4}+\left(p_{4}^{2}-s\right)\left(p_{4}^{2}-s-t\right)-p_{3}^{2}(2 s+t)\right) G_{1,1,1,1,1,1,1,0,-1}
$$

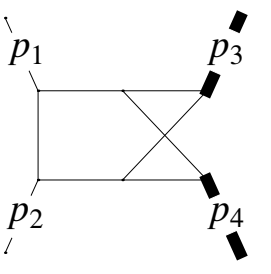

$$
\begin{aligned}
& +\left(2 p_{3}^{2} p_{4}^{2}-p_{3}^{2} t-p_{4}^{2} t+s t\right)\left(G_{0,1,1,1,1,1,1,0,0}+G_{1,0,1,1,1,1,1,0,0}\right. \\
& \left.\left.-G_{1,1,1,1,1,1,1,0,-1}-\left(p_{3}^{2}+p_{4}^{2}-s\right) G_{1,1,1,1,1,1,1,0,0}\right)\right]
\end{aligned}
$$

$$
\begin{aligned}
f_{50}^{\mathrm{N} 34} \sim & -x^{-4 \epsilon}\left(\frac{1}{2}+\frac{2 i \pi \epsilon}{3}-\frac{5 \pi^{2} \epsilon^{2}}{12}-\left(\frac{\zeta_{3}}{2}+\frac{5 i \pi^{3}}{18}\right) \epsilon^{3}+\left(+\frac{53 \pi^{4}}{360}-\frac{i \pi \zeta_{3}}{3}\right) \epsilon^{4}\right) \\
& +x^{-3 \epsilon}\left(1+i \pi \epsilon-\frac{2 \pi^{2} \epsilon^{2}}{3}+\left(2 \zeta_{3}-\frac{i \pi^{3}}{3}\right) \epsilon^{3}+\left(\frac{\pi^{4}}{10}+2 i \pi \zeta_{3}\right) \epsilon^{4}\right) \\
& -x^{-2 \epsilon}\left(\frac{1}{2}-\frac{\pi^{2} \epsilon^{2}}{12}-\frac{7 \epsilon^{3} \zeta_{3}}{2}-\frac{\pi^{4} \epsilon^{4}}{6}\right) \\
& +\frac{5 i \pi \epsilon}{3}\left[4 y^{2}(1-z)\right]^{-3 \epsilon} x^{-4 \epsilon} N_{3}
\end{aligned}
$$

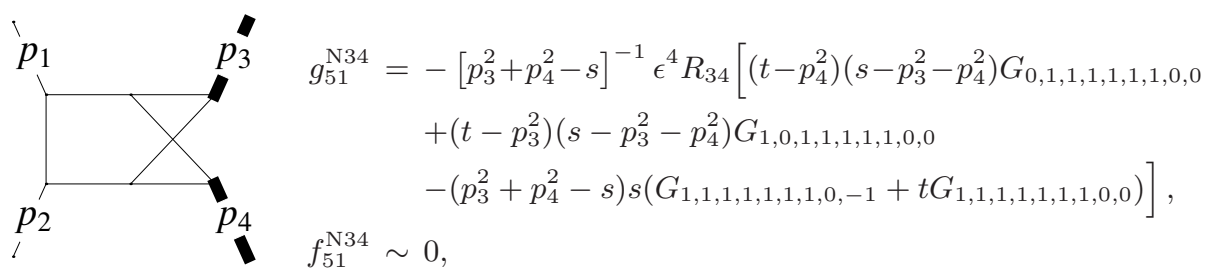


To illustrate how analytic results look like, we provide contributions through $\mathcal{O}\left(\epsilon^{2}\right)$ for three different master integrals. We introduce the following notation

$$
\begin{array}{lll}
a_{1}=z-1, & a_{2}=(1+x) / x, & a_{3}=(1+x(1-z)) / x, \\
a_{4}=\frac{1+y+x y+x y^{2}}{2(1+x) y}, & a_{5}=\frac{1-y-x y+x y^{2}}{2(1+x) y}, & a_{6}=\frac{1+x y}{2 x y} .
\end{array}
$$

For the three integrals that we show below, we separate real and imaginary parts and write

$$
f_{i}^{N I J}=\operatorname{Re} f_{i}^{N I J}+i \operatorname{Im} f_{i}^{N I J},
$$

The explicit expressions read

$$
\begin{aligned}
& \operatorname{Re} f_{33}^{N 12}=-\frac{1}{4}+\epsilon\left[\frac{1}{2} G\left(-\frac{1}{y}, x\right)+\frac{1}{2} G(-1, x)-\frac{1}{2} G(0, y)+G(0, z)\right] \\
& +\epsilon^{2}\left[G(-1, x)(G(0, y)-2 G(0, z))+G(0, y)\left(-2 G\left(-\frac{z}{y}, x\right)+G\left(-\frac{1}{y}, x\right)\right.\right. \\
& +2 G(0, x)-G(0, z)-G(1, z))+G(0, z)\left(2 G\left(-\frac{z}{y}, x\right)-2 G\left(-\frac{1}{y}, x\right)\right. \\
& \left.+2 G\left(-\frac{1}{z}, x\right)+G(z, y)\right)-2 G\left(-\frac{z}{y}, 0, x\right)-G\left(-1,-\frac{1}{y}, x\right)-G\left(-\frac{1}{y},-1, x\right) \\
& -G\left(-\frac{1}{y},-\frac{1}{y}, x\right)-2 G(0, x) G(0, z)+2 G\left(-\frac{1}{z}, 0, x\right)-G(-1,-1, x)+2 G(0,0, x) \\
& +G(1, z) G(z, y)-G\left(0, a_{1}, y\right)-G(z, 0, y)+G\left(z, a_{1}, y\right)+2 G(0,0, y)-G(0,0, z) \\
& \left.+G(0,1, z)+G(1,0, z)+G(1,1, z)-\frac{23 \pi^{2}}{12}\right]+\mathcal{O}\left(\epsilon^{3}\right) \\
& \operatorname{Im} f_{33}^{N 12}=\frac{\pi \epsilon}{2}+\epsilon^{2}\left[2 \pi G\left(-\frac{z}{y}, x\right)-\pi G\left(-\frac{1}{y}, x\right)+2 \pi G\left(-\frac{1}{z}, x\right)-\pi G(-1, x)\right. \\
& -2 \pi G(0, x)+2 \pi G(z, y)-3 \pi G(0, y)+2 \pi G(0, z)+2 \pi G(1, z)]+\mathcal{O}\left(\epsilon^{3}\right) . \\
& \operatorname{Re} f_{33}^{N 13}=\frac{1}{4}-\frac{\epsilon}{2}\left[G\left(-\frac{1}{x}, y\right)+G(-1, x)-2 G(0, x)-G\left(a_{1}, y\right)-G(0, z)-G(1, z)\right] \\
& -\epsilon^{2}\left[G(-1, x) G\left(a_{1}, y\right)+G\left(-\frac{1}{x}, y\right)(-G(-1, x)+2 G(0, x)+G(0, z)+G(1, z))\right. \\
& +G(1, z)\left(-2 G\left(-a_{3}, y\right)+2 G\left(a_{1}, y\right)-G(z, y)\right)-2 G(0, x) G\left(-a_{3}, y\right) \\
& +G\left(-\frac{1}{x}, a_{1}, y\right)+G\left(a_{1},-\frac{1}{x}, y\right)-2 G\left(-a_{3}, a_{1}, y\right)-G\left(-\frac{1}{x},-\frac{1}{x}, y\right) \\
& +G(-1, x) G(1, z)+G(-1, x) G(0, z)-2 G(0, x) G\left(-\frac{1}{x}, z\right)-2 G(0, x) G\left(a_{2}, z\right) \\
& -2 G\left(-\frac{1}{x}, 0, z\right)-2 G\left(a_{2}, 1, z\right)-G(-1,-1, x)+2 G(0,-1, x)-G(0, z) G(z, y) \\
& -G\left(a_{1}, 0, y\right)+2 G\left(a_{1}, a_{1}, y\right)+G(z, 0, y)-G\left(z, a_{1}, y\right)+G(0,0, z)-G(0,1, z) \\
& \left.-G(1,0, z)+G(1,1, z)+3 \pi^{2}\right]+\mathcal{O}\left(\epsilon^{3}\right), \\
& \operatorname{Im} f_{33}^{N 13}=\frac{3 \pi \epsilon}{2}-\epsilon^{2}\left[3 \pi G\left(-\frac{1}{x}, y\right)-2 \pi G\left(-a_{3}, y\right)-\pi G(0, z)-2 \pi G\left(-\frac{1}{x}, z\right)\right. \\
& \left.-2 \pi G\left(a_{2}, z\right)+\pi G(-1, x)-2 \pi G(0, x)+\pi G\left(a_{1}, y\right)-2 \pi G(z, y)-\pi G(1, z)\right]+\mathcal{O}\left(\epsilon^{3}\right) .
\end{aligned}
$$




$$
\begin{aligned}
\operatorname{Re} f_{37}^{N 34}= & \epsilon^{2}\left[-G\left(a_{6}, a_{4}, z\right)+\frac{1}{2} G\left(0, a_{4}, z\right)+\frac{1}{2} G\left(1, a_{4}, z\right)+\frac{1}{2} G\left(0,-a_{5}, z\right)\right. \\
& +\frac{1}{2} G\left(1,-a_{5}, z\right)-G\left(-\frac{1-y}{2 y},-a_{5}, z\right)+G(0, x)\left(G\left(-\frac{1-y}{2 y}, z\right)-G\left(a_{6}, z\right)\right) \\
& +G(-1, y)\left(-G\left(\frac{x y+1}{2 x y}, z\right)+G\left(\frac{1}{y}, x\right)-G(0, x)+G\left(-\frac{1-y}{2 y}, z\right)\right) \\
& +G\left(\frac{1}{y}, x\right)\left(G\left(a_{6}, z\right)-G\left(-\frac{1-y}{2 y}, z\right)\right)+G\left(0, \frac{1}{y}, x\right)+G\left(\frac{1}{y}, 0, x\right) \\
& \left.-G\left(\frac{1}{y}, \frac{1}{y}, x\right)-G(0,0, x)-G(-1,-1, y)-\frac{\pi^{2}}{2}\right], \\
\operatorname{Im} f_{37}^{N 34}= & \epsilon^{2}\left[-\pi G\left(a_{6}, z\right)-\pi G\left(-\frac{1}{y}, x\right)+\pi G(0, x)-\pi G\left(-\frac{1-y}{2 y}, z\right)\right. \\
& +2 \pi G(0, y)-\pi G(1, y)+\pi G(0, z)+\pi G(1, z)+2 \pi \ln 2]
\end{aligned}
$$

As a final comment, we describe some checks of our results. First, as we already mentioned, the boundary conditions for the integrals were obtained using two different methods. Second, we have checked that all computed integrals satisfy the differential equations. Third, many of the integrals that appear in this computation are, in fact, the planar ones. We have recalculated those integrals using the setup that is used for non-planar integrals, including different parametrization for the $N_{34}$ family, and found full agreement with our previous results. We computed some of the integrals numerically using the new version of the program FIESTA [21], that is capable of calculating certain Feynman integrals in the physical region. For all integrals that were computed by FIESTA with sufficient accuracy, agreement with analytic results was found. Finally, we compared the integrals reported in this paper with the results of the recent calculation of two-loop four-point non-planar integrals in the equal mass case, reported recently in ref. [25]; complete agreement was found.

\section{Conclusions}

In this paper we reported on the computation of all two-loop non-planar master integrals that are required to describe production of two off-shell vector bosons in hadron collisions. These integrals were calculated using the differential equations method of ref. [11]. To solve the differential equations, we require boundary conditions. We computed the relevant boundary conditions in the physical region and used them to construct analytic results for non-planar integrals in terms of Goncharov polylogarithms.

The results for the master integrals in terms of Goncharov polylogarithms, as well as the matrices $\tilde{A}$ appearing in the differential equations, are included in the arXiv submission. We note that representation of master integrals in terms of Goncharov polylogarithms may not be the most compact one but it has the advantage that these functions are by now standard and dedicated numerical implementations exist [26, 27]. Also, this representation manifestly separates real and imaginary parts. We did not try to simplify these results, although such simplifications should be possible. Probably the most compact and flexible form can be achieved in terms of Chen iterated integrals [28], at the cost of giving up the 
feature of a linear parametrization. In this spirit, in the recent paper [29] dealing with similar multi-scale integrals, it was shown how a one-dimensional integral representations can be obtained that gives fast and reliable numerical results. Another possibility is to rewrite the results in terms of a minimal function basis (but allowing for more complicated arguments of those functions), which up to weight four consists of classical polylogarithms and one other function, which may be chosen to be $\operatorname{Li}_{2,2}[30,31]$.

Finally, we note that the results presented in this paper provide the last missing ingredient - the non-planar master integrals - for the computation of two-loop amplitudes that describe annihilation of two massless partons into two off-shell gauge bosons. Once these amplitudes become available, theoretical predictions for the production of electroweak gauge bosons at the LHC will be substantially improved.

\section{Acknowledgments}

We would like thank L. Tancredi for providing numerical cross-checks for some of the results reported in this paper. J.M.H. is supported in part by the DOE grant DE-SC0009988 and by the Marvin L. Goldberger fund. The work of F.C. and K.M. is partially supported by US NSF under grant PHY-1214000. K.M. is also supported by Karlsruhe Institute of Technology through its distinguished researcher fellowship program. The work of V.S. was supported by the Alexander von Humboldt Foundation (Humboldt Forschungspreis). We are grateful to the Institute for Theoretical Particle Physics (TTP) at Karlsruhe Institute of Technology where some of the results were obtained. We are indebted to A. Smirnov for the possibility to use his c++ version of FIRE.

Open Access. This article is distributed under the terms of the Creative Commons Attribution License (CC-BY 4.0), which permits any use, distribution and reproduction in any medium, provided the original author(s) and source are credited.

\section{References}

[1] J.M. Henn, K. Melnikov and V.A. Smirnov, Two-loop planar master integrals for the production of off-shell vector bosons in hadron collisions, JHEP 05 (2014) 090 [arXiv: 1402.7078] [INSPIRE].

[2] L.J. Dixon, Z. Kunszt and A. Signer, Helicity amplitudes for $\mathcal{O}\left(\alpha_{s}\right)$ production of $W^{+} W^{-}$, $W^{ \pm} Z, Z Z, W^{ \pm} \gamma$, or $Z \gamma$ pairs at hadron colliders, Nucl. Phys. B 531 (1998) 3 [hep-ph/9803250] [INSPIRE].

[3] L.J. Dixon, Z. Kunszt and A. Signer, Vector boson pair production in hadronic collisions at order $\alpha_{s}$ : lepton correlations and anomalous couplings, Phys. Rev. D 60 (1999) 114037 [hep-ph/9907305] [INSPIRE].

[4] A. Bierweiler, T. Kasprzik and J.H. Kühn, Vector-boson pair production at the LHC to $\mathcal{O}\left(\alpha^{3}\right)$ accuracy, JHEP 12 (2013) 071 [arXiv: 1305.5402] [INSPIRE].

[5] J. Baglio, L.D. Ninh and M.M. Weber, Massive gauge boson pair production at the LHC: a next-to-leading order story, Phys. Rev. D 88 (2013) 113005 [arXiv:1307.4331] [INSPIRE]. 
[6] S. Dawson, I.M. Lewis and M. Zeng, Threshold resummed and approximate next-to-next-to-leading order results for $W^{+} W^{-}$pair production at the $\mathrm{LHC}$, Phys. Rev. D 88 (2013) 054028 [arXiv: 1307. 3249] [INSPIRE].

[7] F. Cascioli et al., Precise Higgs-background predictions: merging NLO QCD and squared quark-loop corrections to four-lepton + 0, 1 jet production, JHEP 01 (2014) 046 [arXiv: 1309.0500] [INSPIRE].

[8] P. Nason and G. Zanderighi, $W^{+} W^{-}, W Z$ and $Z Z$ production in the POWHEG-BOX-V2, Eur. Phys. J. C 74 (2014) 2702 [arXiv:1311.1365] [INSPIRE].

[9] F. Campanario, M. Rauch and S. Sapeta, $W^{+} W^{-}$production at high transverse momenta beyond NLO, Nucl. Phys. B 879 (2014) 65 [arXiv:1309.7293] [INSPIRE].

[10] T. Gehrmann, L. Tancredi and E. Weihs, Two-loop master integrals for $q \bar{q} \rightarrow V V$ : the planar topologies, JHEP 08 (2013) 070 [arXiv:1306.6344] [INSPIRE].

[11] J.M. Henn, Multiloop integrals in dimensional regularization made simple, Phys. Rev. Lett. 110 (2013) 251601 [arXiv:1304.1806] [INSPIRE].

[12] A.V. Kotikov, Differential equations method: new technique for massive Feynman diagrams calculation, Phys. Lett. B 254 (1991) 158 [inSPIRE].

[13] E. Remiddi, Differential equations for Feynman graph amplitudes, Nuovo Cim. A 110 (1997) 1435 [hep-th/9711188] [INSPIRE].

[14] A.V. Smirnov, Algorithm FIRE - Feynman Integral REduction, JHEP 10 (2008) 107 [arXiv:0807.3243] [INSPIRE].

[15] A.V. Smirnov and V.A. Smirnov, FIRE4, LiteRed and accompanying tools to solve integration by parts relations, Comput. Phys. Commun. 184 (2013) 2820 [arXiv:1302.5885] [INSPIRE].

[16] M. Beneke and V.A. Smirnov, Asymptotic expansion of Feynman integrals near threshold, Nucl. Phys. B 522 (1998) 321 [hep-ph/9711391] [INSPIRE].

[17] V.A. Smirnov, Applied asymptotic expansions in momenta and masses, Springer Tracts Mod. Phys. 177 (2002) 1 [INSPIRE].

[18] V.A. Smirnov, Analytic tools for Feynman integrals, Springer Tracts Mod. Phys. 250 (2012) 1 [INSPIRE].

[19] A. Pak and A. Smirnov, Geometric approach to asymptotic expansion of Feynman integrals, Eur. Phys. J. C 71 (2011) 1626 [arXiv:1011.4863] [InSPIRE].

[20] B. Jantzen, A.V. Smirnov and V.A. Smirnov, Expansion by regions: revealing potential and Glauber regions automatically, Eur. Phys. J. C 72 (2012) 2139 [arXiv:1206.0546] [INSPIRE].

[21] A.V. Smirnov, FIESTA 3: cluster-parallelizable multiloop numerical calculations in physical regions, Comput. Phys. Commun. 185 (2014) 2090 [arXiv:1312.3186] [INSPIRE].

[22] J.M. Henn, A.V. Smirnov and V.A. Smirnov, Analytic results for planar three-loop four-point integrals from a Knizhnik-Zamolodchikov equation, JHEP 07 (2013) 128 [arXiv:1306.2799] [INSPIRE].

[23] J.M. Henn, A.V. Smirnov and V.A. Smirnov, Evaluating single-scale and/or non-planar diagrams by differential equations, JHEP 03 (2014) 088 [arXiv:1312.2588] [INSPIRE].

[24] M. Argeri et al., Magnus and Dyson series for master integrals, JHEP 03 (2014) 082 [arXiv: 1401.2979] [INSPIRE]. 
[25] T. Gehrmann, A. von Manteuffel, L. Tancredi and E. Weihs, The two-loop master integrals for $q \bar{q} \rightarrow V V$, JHEP 06 (2014) 032 [arXiv: 1404.4853] [INSPIRE].

[26] C.W. Bauer, A. Frink and R. Kreckel, Introduction to the GiNaC framework for symbolic computation within the C++ programming language, J. Symbol. Comput. 33 (2002) 1 [cs/0004015].

[27] J. Vollinga and S. Weinzierl, Numerical evaluation of multiple polylogarithms, Comput. Phys. Commun. 167 (2005) 177 [hep-ph/0410259] [INSPIRE].

[28] K.-T. Chen, Iterated path integrals, Bull. Amer. Math. Soc. 83 (1977) 831 [INSPIRE].

[29] S. Caron-Huot and J.M. Henn, Iterative structure of finite loop integrals, JHEP 06 (2014) 114 [arXiv: 1404.2922] [INSPIRE].

[30] A.B. Goncharov, M. Spradlin, C. Vergu and A. Volovich, Classical polylogarithms for amplitudes and Wilson loops, Phys. Rev. Lett. 105 (2010) 151605 [arXiv:1006.5703] [INSPIRE].

[31] C. Duhr, H. Gangl and J.R. Rhodes, From polygons and symbols to polylogarithmic functions, JHEP 10 (2012) 075 [arXiv: 1110.0458] [INSPIRE]. 